\title{
Limited data problems for the generalized Radon transform in $\mathbb{R}^{n}$
}

\author{
Jürgen Frikel* and Eric Todd Quinto ${ }^{\dagger}$
}

February 27, 2018

\begin{abstract}
We consider the generalized Radon transform (defined in terms of smooth weight functions) on hyperplanes in $\mathbb{R}^{n}$. We analyze general filtered backprojection type reconstruction methods for limited data with filters given by general pseudodifferential operators. We provide microlocal characterizations of visible and added singularities in $\mathbb{R}^{n}$ and define modified versions of reconstruction operators that do not generate added artifacts. We calculate the symbol of our general reconstruction operators as pseudodifferential operators, and provide conditions for the filters under which the reconstruction operators are elliptic for the visible singularities. If the filters are chosen according to those conditions, we show that almost all visible singularities can be recovered reliably. Our work generalizes the results for the classical line transforms in $\mathbb{R}^{2}$ and the classical reconstruction operators (that use specific filters). In our proofs, we employ a general paradigm that is based on the calculus of Fourier integral operators. Since this technique does not rely on explicit expressions of the reconstruction operators, it enables us to analyze more general imaging situations. medskip
\end{abstract}

Keywords: Radon transforms, Microlocal analysis, Computed tomography, Lambda tomography, Limited angle tomography, Fourier integral operators 2010 AMS Subject Classifications. Primary: 42A12, 93C55, 35S30, Secondary: 65R10, 58J40

\footnotetext{
${ }^{*}$ Department of Applied Mathematics and Computer Science, Technical University of Denmark, Matematiktorvet 303, 2800 Kgs Lyngby, Denmark. Email: jyfr@dtu.dk

${ }^{\dagger}$ Department of Mathematics, Tufts University, Medford, MA 02155, USA. Email: todd.quinto@tufts.edu
} 


\section{Introduction}

In this article, we analyze the limited data problem for the generalized Radon transform integrating over hyperplanes in $\mathbb{R}^{n}$ using microlocal analysis. The integration along those hyperplanes is performed with respect to some weight functions that might depend on both the hyperplane and the point on the hyperplane. By considering more general weight functions, we aim to analyze a wider class of imaging applications, including emission tomography (such SPECT and some models of PET) where the attenuated Radon transform is used to model the measurement process, cf. [12, 13], as well as possible future applications. Of course, our setup also includes the results for the classical Radon transform with constant weight.

Our setup is as follows: Let $(\omega, s) \in \Xi:=S^{n-1} \times \mathbb{R}$, then we consider the hyperplanes

$$
H(\omega, s)=\left\{x \in \mathbb{R}^{n}: x \cdot \omega=s\right\}
$$

perpendicular to $\omega$ containing the point $s \omega$, i.e., $H(\omega, s)$ is $s$ directed units from the origin (in the direction of $\omega$ if $s \geq 0$ and in the opposite direction if $s<0)$. Let $\mu: S^{n-1} \times \mathbb{R}^{n} \rightarrow \mathbb{R}$ be a smooth nowhere zero weight, then we define the generalized Radon transform

$$
R_{\mu} f(\omega, s)=\int_{x \in H(\omega, s)} f(x) \mu(\omega, x) \mathrm{d} x
$$

where $\mathrm{d} x$ is the Lebesgue measure on the hyperplane $H(\omega, s)$. We also define a generalized dual operator (or the backprojection operator) with respect to an arbitrary smooth weight $\nu=\nu(\omega, x)$ as

$$
R_{\nu}^{*} g(x)=\int_{\omega \in S^{n-1}} g(\omega, x \cdot \omega) \nu(\omega, x) \mathrm{d} \omega .
$$

Note that this covers both standard cases when $\nu=\mu$ and so $R_{\nu}^{*}$ is the adjoint operator $\left(R_{\mu}\right)^{*}$ and the case when $\nu=1 / \mu$ which is considered by some authors including Beylkin. We discuss these cases further in Remark 4. Moreover, we note that the above transforms are both defined and weakly continuous for classes of distributions [6].

Many inversion formulas have been proven for the classical Radon transform $(\mu \equiv 1)$ [12], and invertibility of the generalized Radon transform $R_{\mu}$ has been well studied (e.g., $[1,16,19])$. Among the most prominent reconstruction formulas are those of filtered backprojection type [1, 10, 12] which have the following form

$$
B g=R_{\nu}^{*} P g
$$


where $g=R_{\mu} f$ and $P$ is a general pseudodifferential operator that "filters" the data $g=R_{\mu} f$. For example, in case of the classical Radon transform the use of filter $P=1 / 2 \cdot(2 \pi)^{1-n}\left(-\partial^{2} / \partial s^{2}\right)^{\frac{n-1}{2}}$ in (4) leads to an exact reconstruction formula, $f=R^{*} P R f$, which is the basis for the standard filtered backprojection (FBP) algorithm [12]. Another prominent example is the so-called Lambda reconstruction formula (employed in local tomography) which uses the filter $P=1 / 2 \cdot(2 \pi)^{1-n}\left(-\partial^{2} / \partial s^{2}\right)^{n / 2}$ in (4) for $n$ is even. In contrast to the FBP reconstruction operator, the Lambda reconstruction operator is local in even dimensions. However, when $n$ is odd, the FBP reconstruction operator is local itself.

In classical imaging setups the FBP type reconstruction operators (4) are usually applied to full (complete) data. As mentioned above, some of those filters even lead to exact reconstructions if the data are complete. In this paper, we consider the problem of reconstructing $f$ from incomplete data by using reconstructions operators (4) with general filters. More precisely, we assume that $R_{\mu} f$ is given only for directions $\omega$ in a closed subset $A \subset S^{n-1}$ with nontrivial interior. Thus, we deal with the restricted (or limited data) generalized Radon transform defined as

$$
R_{\mu, A}:=\chi_{A \times \mathbb{R}} R_{\mu}
$$

where $\chi_{A \times \mathbb{R}}$ denotes the characteristic function of the data space $A \times \mathbb{R}$ with the limited angular range $A$. Such limited data problems arise in many practical situations and the filtered backprojection type reconstruction of the form (4) is still one of the preferred reconstruction methods [18] (where instead of the full data $g=R_{\mu} f$, the limited data $g_{A}=R_{\mu, A} f$ is used for the reconstruction). It is well known that the limited data reconstruction problem is severely ill-posed [11, 12]. As a consequence, only visible singularities can be reconstructed reliably [20] and additional artifacts can be generated, cf. [2, 9]. In $\mathbb{R}^{2}$, the geometry of added artifacts has been precisely characterized in $[2,9]$. In those articles, the authors consider the classical limited angle FBP and Lambda reconstructions, i.e., $\mu=\nu \equiv 1$ and $P=1 /(4 \pi) \sqrt{-d^{2} / d s^{2}}$ for FBP and $P=(1 / 4 \pi)\left(-d^{2} / d s^{2}\right)$ for Lambda. In [9], Katsevich also considers general weights $\mu$ (and the dual transform w.r.t. weight $1 / \mu$ ) and the Lambda reconstruction operator. In particular, the authors of $[2,9]$ show that artifacts are generated along straight lines that are tangent to singularities of $f$ whose directions correspond to the ends of the angular range. For the classical Radon transform in $\mathbb{R}^{2}$, the strength of added artifacts was characterized by L. Nguyen in [14]. In addition to characterization of artifacts, the authors of $[2,9]$ show that the 
artifacts can be reduced by using modified reconstruction operators. The same modified reconstruction operators are considered in [10] for $R_{\mu}$ with Lambda and FBP filters, and the symbols are given for those specific operators for limited angle and ROI data. In all of those cases, the calculation of the symbols relies on the specific form of the filters.

This work is a generalization of the above mentioned results as it provides a full characterization of visible singularities and added artifacts for the restricted generalized hyperplane Radon transform in $\mathbb{R}^{n}$ and for reconstruction operators with general filters. To prove these characterizations we utilize a general paradigm that is based on the calculus of Fourier integral operators and microlocal analysis. This was originally developed in [3, 4], and in [4] it was used to characterize artifacts in photoacoustic tomography and sonar (see also [15] for related results). This methodology significantly different from techniques used in [2, 9] (which rely on explicit expressions of the reconstruction operators as singular pseudodifferential operators). The flexibility of this approach allows us to prove characterizations for reconstruction operators with general filters as well as general weights.

In the case of $\mathbb{R}^{2}$, our characterization (cf. Corollary 4.1) of visible and added singularities are in accordance with the results of $[2,9]$. However, our result is more general as it is valid for reconstruction operators with general filters and weights, and it provides conditions on filters and weights which guarantee the recoverability of almost all visible singularities. In addition to that, we also prove characterizations in the general case of the hyperplane transform in $\mathbb{R}^{n}$ in Theorem 5.4. To the best of our knowledge this is the first characterization of added artifacts and visible singularities in $\mathbb{R}^{n}$. In this paper, we also define modified versions of the reconstruction operators according to $[2,4,9,10]$ and prove that for general filters $P$ that they do not add artifacts to the reconstruction (Theorem 6.1). Furthermore, we calculate the symbol of our general reconstruction operators as pseudodifferential operators (Theorem 6.1), and provide conditions for filters under which the reconstructions operators are elliptic (Theorem 6.3). If the filters are chosen according to those conditions, we show that classical as well as modified reconstruction operators reliably recover almost all visible singularities (Theorem 5.4 and 6.2, respectively).

The article is organized as follows. Basic definitions and notations are given in Section 2. In Section 3 we present a general paradigm to characterize added singularities in limited data tomography. In Section 4 we first present the characterizations of the limited angle artifacts for the generalized Radon transform in $\mathbb{R}^{2}$. The generalization of these results to $\mathbb{R}^{n}$ is stated in Section 5 , and the artifact reduction strategy as well as symbol calculations are given 
in Section 6. The proofs are presented in the appendix.

\section{Notation}

Let $\Omega$ be an open set. We denote the set of $C^{\infty}$ functions with domain $\Omega$, by $\mathcal{E}(\Omega)$ and the set of $C^{\infty}$ functions of compact support in $\Omega$ by $\mathcal{D}(\Omega)$. Distributions are continuous linear functionals on these function spaces. The dual space to $\mathcal{D}(\Omega)$ is denoted $\mathcal{D}^{\prime}(\Omega)$ and the dual space to $\mathcal{E}(\Omega)$ is denoted $\mathcal{E}^{\prime}(\Omega)$. In fact, $\mathcal{E}^{\prime}(\Omega)$ is the set of distributions of compact support in $\Omega$. For more information about these spaces we refer to [21].

We will use the framework of microlocal analysis for our characterizations. Here, the notion of a wavefront set of a distribution $f \in \mathcal{D}^{\prime}(\Omega)$ is central. It simultaneously describes the locations and directions of singularities of $f$. That is, $f$ has a singularity at $x_{0} \in \Omega$ in direction $\xi_{0} \in \mathbb{R}^{n} \backslash \mathbf{0}$ if for any cutoff function $\varphi$ at $x_{0}$, the Fourier transform $\mathcal{F}(\varphi f)$ does not decay rapidly in any open conic neighborhood of the ray $\left\{t \xi_{0}: t>0\right\}$. Then, the wavefront set of $f \in \mathcal{D}^{\prime}(\Omega), \mathrm{WF}(f)$, is defined as the set of all tuples $\left(x_{0}, \xi_{0}\right)$ such that $f$ is singular at $x_{0}$ in direction $\xi_{0}$. As defined, $\operatorname{WF}(f)$, is a closed subset of $\mathbb{R}^{n} \times\left(\mathbb{R}^{n} \backslash \mathbf{0}\right)$ that is conic in the second variable. However, in what follows, we will view the wavefront set as a subset of a cotangent bundle so it will be invariantly defined on manifolds [22].

We recall that, for a manifold $\Xi$ and $y \in \Xi$, the cotangent space of $\Xi$ at $y, T_{y}^{*}(\Xi)$ is the vector space of all first order differentials (the dual vector space to the tangent space $\left.T_{y}(\Xi)\right)$, and the cotangent bundle $T^{*}(\Xi)$ is the vector bundle with fiber $T_{y}^{*}(\Xi)$ above $y \in \Xi$. That is $T^{*}(\Xi)=$ $\left\{(y, \eta): y \in \Xi, \eta \in T_{y}^{*}(\Xi)\right\}$. The differentials $\mathrm{d} x_{1}, \mathrm{~d} x_{2}, \ldots$, and $\mathrm{d} x_{n}$ are a basis of $T_{x}^{*}\left(\mathbb{R}^{n}\right)$ for any $x \in \mathbb{R}^{n}$. For $\xi \in \mathbb{R}^{n}$, we will use the notation

$$
\xi \mathrm{d} x=\xi_{1} \mathrm{~d} x_{1}+\xi_{2} \mathrm{~d} x_{2}+\cdots+\xi_{n} \mathrm{~d} x_{n} \in T_{x}^{*}\left(\mathbb{R}^{n}\right) .
$$

If $\phi \in \mathbb{R}$ then $\mathrm{d} \phi$ will be the differential with respect to $\phi$, and differentials $\mathrm{d} r$ and $\mathrm{d} s$ are defined analogously.

For the Radon transform in $\mathbb{R}^{n}$, we introduce some more notation. For $\omega \in S^{n-1}$ we define

$$
\pi_{\omega}: \mathbb{R}^{n} \rightarrow H(\omega, 0), \quad \pi_{\omega}(x)=x-(x \cdot \omega) \omega .
$$

So, $\pi_{\omega}(x)$ is the orthogonal projection of $x$ onto this hyperplane. Note that

$$
\pi_{\omega}(x)=\pi_{-\omega}(x) \quad \forall x \in \mathbb{R}^{n} .
$$


Then, $\pi_{\omega}(x) \mathrm{d} \omega$ is the covector in $T_{\omega}^{*}\left(S^{n-1}\right)$ dual to the vector $\pi_{\omega}(x) \in$ $H(\omega, 0)$ (where we have identified this hyperplane with the tangent space $\left.T_{\omega}\left(S^{n-1}\right)\right)$.

Let $X$ and $Y$ be manifolds, and $C \subset T^{*}(Y) \times T^{*}(X)$, then

$$
C^{t}=\{(x, \xi ; y, \eta):(y, \eta ; x, \xi) \in C\} .
$$

If $D \subset T^{*}(X)$, we define

$$
C \circ D=\left\{(y, \eta) \in T^{*}(Y): \exists(x, \xi) \in D:(y, \eta ; x, \xi) \in C\right\} .
$$

Furthermore, if $\Pi_{L}: C \rightarrow T^{*}(Y)$ and $\Pi_{R}: C \rightarrow T^{*}(X)$ are the natural projections, then

$$
C \circ D=\Pi_{L}\left(\Pi_{R}^{-1}(D)\right) .
$$

Fourier integral operators (FIO) are linear operators on distribution spaces that precisely transform wavefront sets. They are defined in [7, 22] in terms of amplitudes and phase functions. If $X$ and $\Xi$ are manifolds and $\mathcal{F}: \mathcal{D}^{\prime}(X) \rightarrow \mathcal{D}^{\prime}(\Xi)$ is a $\mathrm{FIO}$, then associated to $\mathcal{F}$ is the canonical relation $C \subset T^{*}(\Xi) \times T^{*}(X)$. Then the Hörmander-Sato Lemma (e.g., [22, Th. 5.4, p. 461]) asserts for $f \in \mathcal{E}^{\prime}(X)$ that

$$
\mathrm{WF}(\mathcal{F} f) \subset C \circ \mathrm{WF}(f) .
$$

\section{The paradigm}

In this section, we will present a methodology that can be used to prove characterizations of visible singularities and limited view artifacts for a number of tomography problems. In the next section, we will apply them to $R_{\mu}$.

This methodology was originally developed in [3, 4] and in [4] it was used to understand visible and added singularities in limited data photoacoustic tomography and sonar. Denote the forward operator by $\mathcal{M}: \mathcal{E}^{\prime}(\Omega) \rightarrow \mathcal{E}^{\prime}(\Xi)$ and assume $\mathcal{M}$ is a FIO. The object space $\Omega$ is a region to be imaged and the data space $\Xi$ is a space that parameterizes the data. A limited data problem for $\mathcal{M}$ will be a specification of a closed subset $B \subset \Xi$ on which data are given, and in this case, the limited data operator can be written

$$
\mathcal{M}_{B} f=\chi_{B} \mathcal{M}
$$

where $\chi_{B}$ is the characteristic function of $B$ and the product just restricts the data to the set $B$. In the cases we consider, the reconstruction operator is of the form

$$
\mathcal{M}^{*} P \mathcal{M}_{B}
$$


where $\mathcal{M}^{*}$ is an appropriate dual or backprojection operator to $\mathcal{M}$, and this models our reconstruction operator (4).

Our next theorem tells what multiplication by $\chi_{B}$ does to the wavefront set. It is a special case of Theorem 8.2.10 in [8].

Theorem 3.1. Let $u$ be a distribution and let $B$ be a closed subset of $\Xi$ with nontrivial interior. If the non-cancellation condition

$$
\forall(y, \xi) \in \mathrm{WF}(u),(y,-\xi) \notin \mathrm{WF}\left(\chi_{A}\right)
$$

holds, then the product $\chi_{A} u$ can be defined as a distribution. In this case, we have

$$
\mathrm{WF}\left(\chi_{B} u\right) \subset \mathcal{Q}(B, \mathrm{WF}(u)),
$$

where for $W \subset T^{*}(\Xi)$

$$
\begin{array}{r}
\mathcal{Q}(B, W):=\{(y, \xi+\eta): y \in B,[(y, \xi) \in W \text { or } \xi=0] \\
\text { and } \left.\left[(y, \eta) \in \operatorname{WF}\left(\chi_{B}\right) \text { or } \eta=0\right]\right\} .
\end{array}
$$

Note that the condition " $y \in B$ " is not in (14) in Hörmander's theorem, but we include this because $\chi_{B}$ is zero (and so smooth) off of $B$ (this is why we assume $B$ is closed, so that its complement is open). Also, note that the case $\xi=\eta=0$ in the definition of $\mathcal{Q}$ is not allowed since the wavefront set does not include the zero covector.

Our paradigm for proving characterizations for visible and added artifacts is given by the following procedure, cf. [4]:

(a) Confirm the forward operator $\mathcal{M}$ is a FIO and calculate its canonical relation, $C$.

(b) Choose the limited data set $B \subset \Xi$ and calculate $\operatorname{WF}\left(\chi_{B}\right)$.

(c) Make sure the non-cancellation condition (12) holds for $\chi_{B}$ and $\mathcal{M} f$. This can be done in general by making sure it holds for $(y, \xi) \in C$ 。 $\left(T^{*}(\Omega) \backslash \mathbf{0}\right)$.

(d) Calculate $\mathcal{Q}(B, C \circ \mathrm{WF}(f))$.

(e) Calculate $C^{t} \circ \mathcal{Q}(B, C \circ \mathrm{WF}(f))$ to find possible visible singularities and added artifacts using [4, Lemma 3.2]:

$$
\mathrm{WF}\left(\mathcal{M}^{*} P \mathcal{M}_{B} f\right) \subset C^{t} \circ \mathcal{Q}(B, C \circ \mathrm{WF}(f)) .
$$

The paradigm does not provide a lower bound for $\mathrm{WF}\left(\mathcal{M}^{*} P \mathcal{M}_{B} f\right)$ in terms of $\mathrm{WF}(f)$, but we will analyze the operators more completely to provide such a bound. 


\section{Characterization of visible singularities and added artifacts in $\mathbb{R}^{2}$}

In this section, we present a characterization for the line transform $\mathbb{R}^{2}$ which is a special case of the more general results for the hyperplane transform in $\mathbb{R}^{n}$ that will be stated in the next section and proven in the appendix. The statement is simpler in $\mathbb{R}^{2}$. Here, we use a slightly different (more convenient) notation and discuss some implications. The presented characterization generalize the results of $[2,9]$ where the authors consider specific filters.

\subsection{The setup in $\mathbb{R}^{2}$}

To make the presentation simpler, we will parametrize a line in $\mathbb{R}^{2}$ in terms of an angle and a signed distance to the origin, as opposed to the parametrization (1). To that end, we let $s \in \mathbb{R}, \phi \in[0,2 \pi]$ and $\theta(\phi)=(\cos (\phi), \sin (\phi))$ be the unit vector in $S^{1}$ in direction $\phi$ and $\theta^{\perp}(\phi)=(-\sin (\phi), \cos (\phi))$, then $\theta^{\perp}(\phi)$ is perpendicular to $\theta(\phi)$. Let $\Xi=[0,2 \pi] \times \mathbb{R}$, then for each $(\phi, s) \in \Xi$,

$$
L(\phi, s)=\left\{x \in \mathbb{R}^{2}: x \cdot \theta(\phi)=s\right\}
$$

is the line containing $s \theta(\phi)$ and normal to $\theta(\phi)$. We let $\mu(\phi, x)$ be a smooth function on $\mathbb{R} \times \mathbb{R}^{2}$ that is $2 \pi$-periodic in $\phi$. Then, the generalized Radon transform can be written as

$$
R_{\mu} f(\phi, s)=\int_{x \in L(\phi, s)} f(x) \mu(\phi, x) \mathrm{d} x,
$$

where $\mathrm{d} x$ denotes the arc length measure on the line. This transform integrates functions along lines. The corresponding dual transform (or the backprojection operator) for $g \in \mathcal{S}\left(S^{1} \times \mathbb{R}\right)$ and a smooth weight $\nu(\phi, x)$ then takes the form

$$
R_{\nu}^{*} g(x)=\int_{0}^{2 \pi} g(\phi, x \cdot \theta(\phi)) \nu(\phi, x) \mathrm{d} \phi,
$$

which is the integral of $g$ over all lines through $x$ (since for each $\theta(\phi), x \in$ $L(\phi, x \cdot \theta(\phi)))$. As noted in the introduction, these transforms are both defined and weakly continuous for classes of distributions [6].

We consider the limited angle problem, i.e., we consider the data space of the form $[a, b] \times \mathbb{R}$ with the limited angular range $[a, b]$ where $b-a<\pi$ (or $b-a<2 \pi$ if $\mu$ is not symmetric). Note that for $b-a \geq \pi$, every line 
can be parameterized by $\phi \in(a, b)$ although for general $\mu$, the weight might be different on the line $L(\phi, s)$ and $L(\phi+\pi,-s): \mu(\phi, x)$ might not equal $\mu(\phi+\pi, x)$ for all $(\phi, x)$. Thus, we deal with the restricted (or limited angle) generalized Radon transform which we define as

$$
R_{\mu,[a, b]} f(\phi, s)=\chi_{[a, b] \times \mathbb{R}}(\phi, s) \cdot R_{\mu} f(\phi, s),
$$

where $\chi_{[a, b] \times \mathbb{R}}$ denotes the characteristic function of $[a, b] \times \mathbb{R}$.

Remark 1. In order to make clear how the characterization in $\mathbb{R}^{2}$ follows from the more general statement in Theorem 5.4, we would like to point out that (in the general statement) all terms involving $\omega \in S^{n-1}$ can be stated in $\mathbb{R}^{2}$ using the parametrization of $\omega=\theta(\phi) \in S^{1}$ with respect to the angle $\phi \in[0,2 \pi]$ (see section 4.1). Hence, all terms that are generally formulated with respect to $\mathrm{d} \omega$ will be stated with respect to $\mathrm{d} \phi$. For example, the projection $\pi_{\omega}$ (cf. (5)) simplifies to $\pi_{\theta(\phi)}(x)=\left(x \cdot \theta^{\perp}(\phi)\right) \theta^{\perp}(\phi)$ and, hence, $\pi_{\omega}(x) \mathrm{d} \omega$ corresponds to $x \cdot \theta^{\perp}(\phi) \mathrm{d} \phi$.

\subsection{The characterization}

The corollary presented below is a special case of Theorem 5.4 that is given in Section 5 and proven in the appendix. The statement is simpler in $\mathbb{R}^{2}$ and the characterizations are in accordance with the results in [2, 9]. However, the following result is more general than the results of $[2,9]$ as it is valid for reconstruction operators with general filters and weights, and it provides a lower bound (24) under an ellipticity assumption.

To state the result in $\mathbb{R}^{2}$, for $A \subset[0,2 \pi]$, we define

$$
V_{A}^{R}=\{(\phi, s, \alpha[-z \mathrm{~d} \phi+\mathrm{d} s]): \phi \in A, s \in \mathbb{R}, z \in \mathbb{R}, \alpha \neq 0\}
$$

and let

$$
\mathcal{V}_{A}=\left\{(x, \alpha \theta(\phi) \mathrm{d} x): x \in \mathbb{R}^{2}, \alpha \neq 0, \phi \in A\right\} .
$$

Now, for $f \in \mathcal{E}^{\prime}\left(\mathbb{R}^{n}\right)$, define

$$
\mathrm{WF}_{A}(f)=\mathrm{WF}(f) \cap \mathcal{V}_{A} .
$$

Similar definitions will be given for the transform in $\mathbb{R}^{n}$, and they will reflect the notation used in that general case.

Corollary 4.1. Let $\mu$ and $\nu$ be smooth $2 \pi$-periodic functions on $\mathbb{R} \times \mathbb{R}^{2}$. Let $P$ be a pseudodifferential operator on $\mathcal{E}^{\prime}(\Xi)$. Let $f \in \mathcal{E}^{\prime}\left(\mathbb{R}^{2}\right)$. Our limited data reconstruction operator is

$$
\mathcal{L}_{[a, b]} f=R_{\nu}^{*} P R_{\mu,[a, b]} f=R_{\nu}^{*} P \chi_{[a, b]} R_{\mu} f .
$$


Then,

$$
\mathrm{WF}\left(\mathcal{L}_{[a, b]} f\right) \subset \mathrm{WF}_{[a, b]}(f) \cup \mathcal{A}_{\{a, b\}}(f),
$$

where $\mathrm{WF}_{[a, b]}(f)$ is defined according to (21) and

$$
\begin{array}{r}
\mathcal{A}_{\{a, b\}}(f)=\left\{\left(x+t \theta^{\perp}(\phi), \alpha \theta(\phi) \mathrm{d} x\right): \phi \in\{a, b\},\right. \\
\alpha, t \neq 0,(x, \alpha \theta(\phi)) \in \mathrm{WF}(f)\}
\end{array}
$$

is the set of possible added artifacts.

Now, assume that $\mu$ and $\nu$ are nowhere zero and $b-a<\pi$. Assume the top order symbol of $P$ is elliptic on $V_{[a, b]}^{R}$ defined by (19). Then,

$$
\mathrm{WF}_{(a, b)}(f)=\mathrm{WF}_{(\mathrm{a}, \mathrm{b})}\left(\mathcal{L}_{[a, b]} f\right) .
$$

The condition $b-a<\pi$ is reasonable in limited data problems because, if $b-a>\pi$, then every line can be parameterized by $L(\phi, s)$ for some $\phi \in(a, b)$ which amounts to the full data problem.

Corollary 4.1 provides an upper bound for the singularities of limited angle reconstructions: It is given as a union of the visible wavefront set of $f, \mathrm{WF}_{[a, b]} f$ (singularities that actually belong to $f$ ), and the set of possibly added artifacts $\mathcal{A}_{\{a, b\}}(f)$ (singularities that might be artificially created by the reconstruction operators). In particular, it shows that any singularity of $f$ with direction outside the given angular range $[a, b]$ is smoothed by the limited angle reconstruction operator $\mathcal{L}_{[a, b]}$. Those singularities cannot be reconstructed (are invisible). The corollary also provides a precise geometric description of possibly added artifacts. It shows that artifacts are generated along straight lines that are normal to singularities of $f$ (e.g., tangent to boundaries of regions) whose directions correspond to the ends of the angular range $\{a, b\}$. In other words, any singularity of $f$ with direction $\theta(a)$ or $\theta(b)$, generates added singularities along the line $L(a, x \cdot \theta(a))$ or $L(b, x \cdot \theta(b))$, respectively.

Moreover, Corollary 4.1 provides a lower bound in form of the equation (24) under an ellipticity assumption. In particular, the equality (24) guarantees that almost all visible singularities will be reconstructed if the filters are chosen appropriately. This statement is formulated for the case $b-a<\pi$ (limited angular range).

\section{Generalizations to the Radon transform in $\mathbb{R}^{n}$}

In this section, we present characterizations of visible singularities and added artifacts for the restricted generalized Radon (hyperplane) transform in $\mathbb{R}^{n}$. 
We analyze filtered backprojection type operators with general filters and derive conditions for filters which guarantee the recoverability of most of the visible singularities. Our results generalize the characterizations given in $[2,9]$. We employ the paradigm introduced in Section 3. In what follows, we use the notation introduced in Section 1 and Section 2.

\subsection{Basic properties of $R_{\mu}$}

The first proposition provides the canonical relation of $R_{\mu}$ and $R_{\nu}^{*}$ (by transpose), and this determines their microlocal properties.

Proposition 5.1. If $\mu$ is smooth weight on $S^{n-1} \times \mathbb{R}^{n}$, then the generalized Radon transform $R_{\mu}$ is a Fourier integral operator associated to the canonical relation

$$
\begin{aligned}
C=\left\{\left((\omega, s), \alpha\left[-\pi_{\omega}(x) \mathrm{d} \omega+\mathrm{d} s\right] ; x, \alpha \omega \mathrm{d} x\right):\right. & \\
\omega & \left.\in S^{n-1}, \alpha \neq 0, x \cdot \omega=s\right\},
\end{aligned}
$$

and $(\omega, x, \alpha)$ give coordinates on $C$ because $s=\omega \cdot x$. If $\mu$ is nowhere zero, then $R_{\mu}$ is elliptic.

If $\nu$ is smooth, then the backprojection operator $R_{\nu}^{*}$ is a Fourier integral operator associated to the canonical relation $C^{t}$ defined in (6), and if $\nu$ is nowhere zero, then $R_{\nu}^{*}$ is elliptic.

Let $\Pi_{R}: C \rightarrow T^{*}\left(\mathbb{R}^{n}\right)$ and $\Pi_{L}: C \rightarrow T^{*}(\Xi)$ be the natural projections. Then $\Pi_{L}$ is an injective immersion and $\Pi_{R}$ is a two-to-one immersion. Let $(x, \xi \mathrm{d} x) \in T^{*}\left(\mathbb{R}^{n}\right) \backslash \mathbf{0}$. Define

$$
\begin{aligned}
\omega(\xi) & =\xi /\|\xi\| \in S^{n-1} \\
\lambda_{0}(x, \xi) & =\left(\omega(\xi), x \cdot \omega(\xi),\|\xi\|\left[-\pi_{\omega}(x) \mathrm{d} \omega+\mathrm{d} s\right]\right) \\
\lambda_{1}(x, \xi) & =\left(-\omega(\xi),-x \cdot \omega(\xi),-\|\xi\|\left[-\pi_{\omega}(x) \mathrm{d} \omega+\mathrm{d} s\right]\right)
\end{aligned}
$$

where $\pi_{\omega}$ is defined by (5).

The two preimages of $(x, \xi \mathrm{d} x)$ under $\Pi_{R}$ are

$$
\left(\lambda_{0}(x, \xi) ; x, \xi \mathrm{d} x\right) \quad \text { and } \quad\left(\lambda_{1}(x, \xi) ; x, \xi \mathrm{d} x\right) .
$$

Therefore,

$$
\begin{aligned}
C \circ\{(x, \xi \mathrm{d} x)\} & =\left\{\lambda_{0}(x, \xi), \lambda_{1}(x, \xi)\right\} \\
C^{t} \circ\left\{\lambda_{0}(x, \xi \mathrm{d} x)\right\} & =C^{t} \circ\left\{\lambda_{1}(x, \xi)\right\}=\{(x, \xi \mathrm{d} x)\} .
\end{aligned}
$$


Proof. The calculation of $C$ is well known, see e.g., [5, 19]. The generalized hyperplane transform $R_{\nu}$ has the same canonical relation as $R_{\mu}$ since the weight does not affect the canonical relation, only the symbol. Since $R_{\nu}^{*}$ is the dual of $R_{\nu}$, it is an FIO associated to $C^{t}$ by the standard calculus of FIO, e.g., [7, Theorem 4.2.1]. That $\Pi_{L}: C \rightarrow T^{*}(\Xi) \backslash \mathbf{0}$ is an injective immersion (The Bolker Assumption) is a straightforward calculation [5, 19].

One uses (25) to find the two preimages of $(x, \xi \mathrm{d} x)$ under $\Pi_{R}: C \rightarrow$ $T^{*}\left(\mathbb{R}^{n}\right) \backslash \mathbf{0}$ using the fact that $\xi=\|\xi\| \omega(\xi)=-\|\xi\|(-\omega(\xi))$. Statement (27) follows from the observation that, if $A \subset T^{*}\left(\mathbb{R}^{n}\right)$, then $C \circ A=\Pi_{L}\left(\Pi_{R}^{-1}(A)\right)$, and if $B \subset T^{*}(\Xi)$, then (8) can be used to show that $C^{t} \circ B=\Pi_{R}\left(\Pi_{L}^{-1}(B)\right)$ (where $\Pi_{R}$ and $\Pi_{L}$ are the maps for $C$ ).

\subsection{The limited data operators}

We are concerned with the limited data problem when $R_{\mu} f$ is given only for angles $\omega$ in a closed subset, $A$, of $S^{n-1}$ with nontrivial interior. The limited data set from Section 3 is

$$
B=A \times \mathbb{R} .
$$

We assume $A$ has nontrivial interior so that $\chi_{A \times \mathbb{R}}$ is not the zero distribution.

Our next proposition shows that the limited data forward operator and our reconstruction operator are defined for distributions.

Proposition 5.2. Let $\mu$ and $\nu$ be smooth functions on $S^{n-1} \times \mathbb{R}^{n}$. Let $P$ be a pseudodifferential operator on $\mathcal{E}^{\prime}(\Xi)$ and let $A \subset S^{n-1}$ be a closed set with nontrivial interior. Let $f \in \mathcal{E}^{\prime}\left(\mathbb{R}^{n}\right)$. Then, the limited data forward operator for data on $A \times \mathbb{R}$,

$$
R_{\mu, A}:=\chi_{A \times \mathbb{R}} R_{\mu}
$$

maps $\mathcal{E}^{\prime}\left(\mathbb{R}^{n}\right)$ to $\mathcal{E}^{\prime}(\Xi)$.

The limited data reconstruction operator

$$
\mathcal{L}_{A}:=R_{\nu}^{*} P R_{\mu, A}
$$

maps $\mathcal{E}^{\prime}\left(\mathbb{R}^{n}\right)$ to $\mathcal{D}^{\prime}\left(\mathbb{R}^{n}\right)$. Here $R_{\nu}^{*}$ is defined by $(3)$.

This proposition will be proven in the appendix as a part of the proof of Theorem 5.4.

To describe the ellipticity conditions in our theorems, we need to define the following sets.

Definition 5.3. Let $A \subset S^{n-1}$. Define

$$
V_{A}^{R}=\{(\omega, s, \alpha[-z \mathrm{~d} \omega+\mathrm{d} s]): \omega \in A, s \in \mathbb{R}, z \in H(\omega, 0), \alpha \neq 0\}
$$


and let

$$
\mathcal{V}_{A}=\left\{(x, \alpha \omega \mathrm{d} x): x \in \mathbb{R}^{n}, \alpha \neq 0, \omega \in A\right\} .
$$

Now, for $f \in \mathcal{E}^{\prime}\left(\mathbb{R}^{n}\right)$, define

$$
\mathrm{WF}_{A}(f)=\mathrm{WF}(f) \cap \mathcal{V}_{A} .
$$

In the next section, we will show that, if $P$ is elliptic on $V_{A}^{R}$, then our reconstruction operator will recover almost all visible singularities, and we will prove that the set $\mathcal{V}_{A}$ will contain singularities of the object that are visible in the data $R_{\mu} f$.

\subsection{The characterization}

The next theorem provides a characterization of visible singularities and added artifacts in arbitrary dimensions (using reconstruction operators with arbitrary filters $P$ ), and it also provides a lower bound (36) under an ellipticity assumption. To state the result, we again let $A \subset S^{n-1}$ and we define

$$
(-1) A=\left\{\omega \in S^{n-1}:-\omega \in A\right\} .
$$

Note that $\operatorname{int}(A)$ is the interior of $A, \operatorname{bd}(A)$ is its boundary, and $\operatorname{cl}(A)$ is its closure in $S^{n-1}$.

Theorem 5.4. Let $\mu$ and $\nu$ be a smooth functions on $S^{n-1} \times \mathbb{R}^{n}$. Let $P$ be a pseudodifferential operator on $\mathcal{E}^{\prime}(\Xi)$. Let $A \subset S^{n-1}$ be a closed set with nontrivial interior and let $\mathcal{L}_{A}$ be defined by (29). Then,

$$
\mathrm{WF}\left(\mathcal{L}_{A} f\right) \subset \mathrm{WF}_{A}(f) \cup \mathcal{A}_{\mathrm{bd}(A)}(f),
$$

where $\mathrm{WF}_{\mathrm{A}}(f)$ is defined in (32) and

$$
\begin{gathered}
\mathcal{A}_{\mathrm{bd}(A)}(f)=\{(x+t y, \alpha \omega \mathrm{d} x): \omega \in \operatorname{bd}(A), t \neq 0, \alpha \neq 0, \\
(x, \alpha \omega \mathrm{d} x) \in \operatorname{WF}(f), y \in H(\omega, 0), \\
\text { and } \left.(\omega, y \mathrm{~d} \omega) \in \operatorname{WF}\left(\chi_{A}\right)\right\}
\end{gathered}
$$

is the set of possible added artifacts.

Now, assume that $\mu$ and $\nu$ are both strictly positive and the top order symbol of $P$ is elliptic on $V_{A}^{R}$ defined by (30). Assume either

(i) the following non-symmetry condition holds:

$$
\forall \omega \in A, \quad-\omega \notin A
$$

or 
(ii) the symbol of $P$ is real and is either always positive or always negative on $V_{A}^{R}$ and $A$ is symmetric (that is $A=(-1) A$ ).

Then,

$$
\mathrm{WF}_{\operatorname{int}(A)}(f)=\mathrm{WF}_{\operatorname{int}(A)}\left(\mathcal{L}_{A} f\right) .
$$

Note that, either the non-symmetry condition (35) or the symmetry condition in (ii) on $A$ is needed. To see this, assume there is a vector $\omega_{0} \in$ $\operatorname{int}(A)$ for which $-\omega_{0} \in \operatorname{bd}(A)$. Then, an added artifact caused by a covector $\left(x,(-\alpha)\left(-\omega_{0}\right) \mathrm{d} x\right) \in \mathrm{WF}(f)$ and a singularity at $\left(-\omega_{0}, y \mathrm{~d} \omega\right) \in \mathrm{WF}\left(\chi_{A}\right)$ can create added artifacts at points $(x+t y, \alpha \omega \mathrm{d} x)$.

The proofs of this and the other main theorems are in the appendix.

Remark 2. This theorem provides both upper and lower bounds for the singularities that can be reconstructed at the limited angular range $\omega \in A$. The upper bound is given as the union of the set of visible singularities $\mathrm{WF}_{A}(f)$ and added artifacts $\mathcal{A}_{\mathrm{bd}(A)}(f)$ by (33). This shows that if a singularity of $f$ is not in $\mathcal{V}_{A}$, then it is smoothed by $\mathcal{L}_{A}$. This is reflected by the fact that $\mathrm{WF}\left(R_{\mu, A} f\right) \subset V_{A}^{R}$, which can be proven using Proposition 5.1.

The lower bound is given by the equality (36) under an ellipticity assumption. In particular, the equality (36) provides a guarantee that almost all visible singularities will be reconstructed if the filters are chosen appropriately.

Remark 3. Radon transforms detect singularities conormal to the set being integrated over (e.g., $[5,17,20]$ ), and the above theorem states this relation explicitly: only singularities $(x, \alpha \omega \mathrm{d} x) \in \mathrm{WF}(f)$ with directions in the visible angular range, $\omega \in A$ (i.e., in $\mathcal{V}_{A}$ ) can be reconstructed from limited data. Singularities of $f$ at covectors outside $\mathcal{V}_{A}$ are smoothed.

The added singularities occur in the following way. Each singularity of $f$ in a direction $\omega \in \operatorname{bd}(A)$ is spread along one or more lines. If $(x, \alpha \omega \mathrm{d} x) \in$ $\mathrm{WF}(f)$, then singularities are spread in the hyperplane $H(\omega, x \cdot \omega)$. For each $y \in H(\omega, 0)$ with $(\omega, y \mathrm{~d} \omega) \in \mathrm{WF}\left(\chi_{A}\right)$, singularities are spread along the line in $H(\omega, x \cdot \omega)$ through $x$ and parallel to $y$. So, if $\operatorname{bd}(A)$ is smooth at $\omega$, then there is only one line of singularities (because the only singularities come from vectors $y$ that are normal to $\operatorname{bd}(A)$ at $\omega)$.

However, if $\operatorname{bd}(A)$ is not smooth at $\omega$, then the singularity at $(x, \alpha \omega \mathrm{d} x)$ is spread on other lines. For example, if $\operatorname{bd}(A)$ has a corner, then for every $y \in H(\omega, 0) \backslash \mathbf{0},(\omega, y \mathrm{~d} \omega) \in \mathrm{WF}\left(\chi_{A}\right)$ so singularities are spread along the whole hyperplane $H(\omega, x \cdot \omega)$. 


\section{Reduction of artifacts and preservation of visi- ble singularities}

In previous sections we have shown that the added artifacts are generated due to the hard truncation at the boundary of the angular range in the limited data generalized Radon transform (18) and (28), respectively. In this section, we define a modified version of the reconstruction operators according to $[2,4,9,10]$ that replace the sharp cutoff $\chi_{A \times \mathbb{R}}$ by a smooth cutoff and we prove that for general filters $P$ they do not add artifacts to the reconstruction.

Let $\varphi$ be a smooth cutoff function supported in $A$. We replace $\chi_{A \times \mathbb{R}}$ by $\varphi$ in the reconstruction operator and define the modified (artifact-reduced) reconstruction operator as

$$
\mathcal{L}_{\varphi} f=R_{\nu}^{*} P \mathcal{K}_{\varphi} R_{\mu} f \text { where } \mathcal{K}_{\varphi} g(\omega, s)=\varphi(\omega) g(\omega, s)
$$

(and where $\mu$ and $\nu$ are smooth weights). This method was analyzed for the line transform in $\mathbb{R}^{2}$ and for the lambda filter $P=-d^{2} / d s^{2}$ and the FBP filter $P=\sqrt{-d^{2} / d s^{2}}$ and with $R_{1}$ in [2] and with $R_{\mu}$ (and backprojection $\left.R_{1 / \mu}^{*}\right)$ in $[9,10]$. Our theorems provide generalization to $\mathbb{R}^{n}$ and to arbitrary filters $P$, and they provide the symbol of $\mathcal{L}_{\varphi}$ in general.

Theorem 6.1. Let $\mu$ and $\nu$ be smooth weights and let $\varphi$ be a smooth function supported in A. Then

$$
\mathrm{WF}\left(\mathcal{L}_{\varphi}(f)\right) \subset \mathrm{WF}_{A}(f) .
$$

The top order symbol of $\mathcal{L}_{\varphi}$ is

$$
\begin{aligned}
\sigma\left(\mathcal{L}_{\varphi}\right)(x, \xi \mathrm{d} x)=\frac{(2 \pi)^{n-1}}{\|\xi\|^{n-1}}\left[\varphi(\omega(\xi)) p\left(\lambda_{0}(x, \xi)\right) \nu(\omega(\xi), x) \mu(\omega(\xi), x)\right. \\
\left.\quad+\varphi(-\omega(\xi)) p\left(\lambda_{1}(x, \xi)\right) \nu(-\omega(\xi), x) \mu(-\omega(\xi), x)\right]
\end{aligned}
$$

where $p$ is the top order symbol of the pseudodifferential operator $P$ and the other notation is given in (26).

The proofs of this and the other main theorems are in the appendix.

The containment (38) shows that the modified reconstruction operators reconstruct only visible singularities and, hence, do not add artifacts. This result provides an upper bound for the visible singularities that are reconstructed through the modified reconstruction operators $\mathcal{L}_{\varphi}$. In the following theorem, we also establish a lower bound for the visible singularities under an ellipticity assumption on the reconstruction operators. 
Remark 4. We now discuss two special weights. If $\nu=\mu$, then $R_{\nu}^{*}$ is the formal adjoint of $R_{\mu}$ and $\nu(\omega(\xi), x) \mu(\omega(\xi), x)$ is replaced by $\mu^{2}(\omega(\xi), x)$ in the symbol of $\mathcal{L}_{\varphi}$.

If $\mu$ is nowhere zero and $\nu=1 / \mu$, then the symbol of $\mathcal{L}_{\varphi}$ is especially simple:

$$
\sigma\left(\mathcal{L}_{\varphi}\right)(x, \xi \mathrm{d} x)=\frac{(2 \pi)^{n-1}}{\|\xi\|^{n-1}}\left[\varphi(\omega(\xi)) p\left(\lambda_{0}(x, \xi)\right)+\varphi(-\omega(\xi)) p\left(\lambda_{1}(x, \xi)\right)\right],
$$

and so the top order symbol of $\mathcal{L}_{\varphi}$ is not affected by the weight $\mu$ and the only $x$ dependence comes from the choice of $P$, as opposed to the general case with arbitrary $\mu$ and $\nu$.

Theorem 6.2. Let $\varphi$ be a nonnegative smooth function supported on $A$ and nonzero on $\operatorname{int}(A)$ and let $\mu$ and $\nu$ be smooth positive weights. Assume the symbol $\sigma\left(\mathcal{L}_{\varphi}\right)$ in $(39)$ is elliptic on $\mathcal{V}_{\operatorname{int}(A)}$ defined in (31). Then,

$$
\mathrm{WF}_{\operatorname{int}(A)}(f) \subset \mathrm{WF}\left(\mathcal{L}_{\varphi}(f)\right) \subset \mathrm{WF}_{A}(f) .
$$

This implies that

$$
\mathrm{WF}_{\operatorname{int}(A)}(f)=\mathrm{WF}_{\operatorname{int}(A)}\left(\mathcal{L}_{\varphi}(f)\right) .
$$

This theorem shows that, as long as the filter $P$ is well-chosen, almost all visible wavefront directions (those in $\mathrm{WF}_{\text {int }(A)}(f)$ ) are visible using the artifact reduced operator $\mathcal{L}_{\varphi}$ and artifacts are not added since $\mathrm{WF}\left(\mathcal{L}_{\varphi}(f)\right)$ is contained in $\mathrm{WF}_{A}(f)$.

Our next theorem provides conditions on the filters $P$ that guarantee the ellipticity of the reconstruction operators $\mathcal{L}_{\varphi}$. In particular, it specifies some cases in which Theorem 6.2 can be applied.

Theorem 6.3. Let $\varphi$ be a nonnegative function supported in $A$ and nonzero on $\operatorname{int}(A)$. Assume that $\mu$ and $\nu$ are smooth and strictly positive and the top order symbol of $P$ is elliptic on $V_{A}^{R}$ defined by (30). Assume either

(i) The following non-symmetry condition holds:

$$
\forall \omega \in A, \quad-\omega \notin A,
$$

(ii) or the symbol of $P$ is real and either always positive or always negative on $V_{A}^{R}$.

Then $\mathcal{L}_{\varphi}=R_{\nu}^{*} \mathcal{K}_{\varphi} P R_{\mu}$ is elliptic on $\mathcal{V}_{\operatorname{int}(A)}$ (defined by (31)). Therefore, (40) and (41) hold. 
Note that condition (42) is the same condition, (35), used in Theorem 5.4 ,

Example 1. We now discuss these conditions for the Radon line transform in the plane and for $\mu=\nu \equiv 1$.

First, we consider two standard filters, $P$. Condition (ii) holds, for example, if $P=-d^{2} / d s^{2}$, the filter in Lambda tomography, or $P=\sqrt{-d^{2} / d s^{2}}$, the filter in FBP because, in both cases, the symbol is of the same sign on $V_{A}^{R}$ (e.g., $\sigma\left(\sqrt{-d^{2} / d s^{2}}\right)(\omega, s, \beta \mathrm{d} \omega+\alpha \mathrm{d} s)=|\alpha|$ is real and never zero), and our theorem can be applied to these operators.

Now, let $P=d / d s$. If $A=\{(\cos (\phi), \sin (\phi)): \phi \in[a, b]\}$ and $b-a<\pi$, then condition (i) holds. Since the symbol of $d / d s$ is nowhere zero on $V_{A}^{R}$, $\mathcal{L}_{\varphi}$ is elliptic on $\mathcal{V}_{\operatorname{int}(A)}$.

However, if $b-a>\pi$, ellipticity of $P$ is not sufficient for ellipticity of $\mathcal{L}_{\varphi}$. For example, consider the full data problem for the classical transform $R_{1}$ and $P=(-i) d / d s$, then $\sigma(P)(\omega, s, \beta \mathrm{d} \omega+\alpha \mathrm{d} s)=\alpha$ changes sign on $V_{A}^{R}$, even though $P$ is elliptic, and the operator $R_{1}^{*}\left((-i) d / d s R_{1}\right)=0$ by symmetry. Of course, the analogous point can be made for $P=d / d s$.

\section{Concluding remarks}

In this work, we have characterized visible singularities and added artifacts for the limited data problem associated with the restricted generalized Radon (hyperplane) transform in $\mathbb{R}^{n}$. In particular, we analyzed filtered backprojection reconstruction operators with general filters and proved that a simple modification of these operators leads to an artifact reduction. To the best of our knowledge this work for the first time provides characterizations of artifacts for the restricted generalized Radon transform in $\mathbb{R}^{n}$ (Theorem 5.4), which includes the classical setup in $\mathbb{R}^{2}$ as a special case (Corollary 4.1). However, even in the case of $\mathbb{R}^{2}$ our results are more general than the characterizations presented in $[2,9]$ since they are valid for general reconstruction operators with arbitrary filters. Our proofs use the general paradigm (originally developed in $[3,4]$ ) that is based on the calculus of Fourier integral operators and microlocal analysis. This technique is substantially different from the one used in $[2,9]$ where the authors use explicit expressions of the considered (specific) reconstruction operators (and hence they know the symbols of these operators explicitly). We would like to note that the paradigm that we use in our proofs does not provide a way to characterize which part of the visible singularities will be reconstructed, it enables us to derive only an upper bound for the wavefront set of the 
limited data reconstructions. In fact, no lower bound can be derived for general reconstruction operators with arbitrary filters. To guarantee that most of the visible singularities will be reconstructed we need to make sure that the reconstruction operators are elliptic. This can be done by choosing the filters appropriately. As one of our main results, in Theorem 6.3, we derive conditions for filters that guarantee ellipticity of the filtered backprojection reconstruction operators. To that end, we calculate the symbol of the general reconstruction operators in Theorem 6.1 .

\section{Acknowledgments}

The authors thank Frank Filbir for encouraging this collaboration. They thank Adel Faridani and Alexander Katsevich for pointing out important references. They also thank Venkateswaran Krishnan for valuable comments concerning this work. They first author acknowledges support from the HC Ørsted Postdoc programme, co-funded by Marie Curie Actions. The work of the second author is partially supported by NSF grant DMS 1311558 .

\section{A Appendix}

We prove our main theorems in this appendix because the proofs are all related.

Proof of Proposition 5.2 and Theorem 5.4. We use the paradigm presented in Section 3 to prove (33). By Proposition 5.1, we know that $R_{\mu}$ is a Fourier integral operator with the canonical relation given in (25). Thus, the step (a) of our paradigm is carried out.

For the step (b), we consider $B=A \times \mathbb{R}$ and compute $\operatorname{WF}\left(\chi_{A \times \mathbb{R}}\right)$. Note that

$$
\mathrm{WF}\left(\chi_{A \times \mathbb{R}}\right)=\left\{(\omega, s ; \eta+0 \mathrm{~d} s):(\omega, \eta) \in \mathrm{WF}\left(\chi_{A}\right), s \in \mathbb{R}\right\} .
$$

Note that $\chi_{A}$ is smooth on the complement of $\mathrm{bd}(A)$, so the covectors in $\mathrm{WF}\left(\chi_{A \times \mathbb{R}}\right)$ all have $\omega \in \operatorname{bd}(A)$.

First, note that every covector in $C \circ\left(T^{*}\left(\mathbb{R}^{n}\right)\right)$ has nonzero $\mathrm{d} s$ component by (25). Therefore, every covector in $\mathrm{WF}\left(R_{\mu} f\right)$ has nonzero d $s$ component. Since $\mathrm{WF}\left(\chi_{A \times \mathbb{R}}\right)$ has zero $\mathrm{d} s$-component, we see that the non-cancellation condition (12) holds. This is step (c) of our paradigm. Hence, by Theorem 3.1, the product $R_{\mu, A} f=\chi_{A \times \mathbb{R}} R_{\mu} f$ is well-defined as a distribution with compact support since $f \in \mathcal{E}^{\prime}\left(\mathbb{R}^{n}\right)$. This proves that $R_{\mu, A}: \mathcal{E}^{\prime}\left(\mathbb{R}^{n}\right) \rightarrow \mathcal{E}^{\prime}(\Xi)$, 
the first statement in Proposition 5.2. Since $P: \mathcal{E}^{\prime}(\Xi) \rightarrow \mathcal{D}^{\prime}(\Xi)$ and $R_{\nu}^{*}$ : $\mathcal{D}^{\prime}(\Xi) \rightarrow \mathcal{D}^{\prime}\left(\mathbb{R}^{n}\right)$, the final statement of that Proposition also holds.

Continuing the proof of Theorem 5.4, we do the next step, (d), of the paradigm, and calculate $\mathcal{Q}(A \times \mathbb{R}, C \circ \mathrm{WF}(f))$ using (14). By definition, the set $\mathcal{Q}(A \times \mathbb{R}, C \circ \mathrm{WF}(f))$ is a union of three sets:

$$
\begin{gathered}
\mathcal{Q}(A \times \mathbb{R}, C \circ \mathrm{WF}(f)) \\
=\left[(C \circ \mathrm{WF}(f)) \cap\left\{((\omega, s), \eta) \in T^{*}(\Xi): \omega \in A\right\}\right] \\
\cup \operatorname{WF}\left(\chi_{A \times \mathbb{R}}\right) \cup W_{\mathrm{bd}(A)}(f),
\end{gathered}
$$

where the first set (in braces) corresponds to $\xi \neq 0, \eta=0$ in the expression " $(y, \xi+\eta)$ " in the definition of $\mathcal{Q},(14)$, the second to $\xi=0, \eta \neq 0$ and the third, $W_{\mathrm{bd}(A)}(f)$, corresponds to $\xi \neq 0, \eta \neq 0$.

Note that points in this third set, $W_{\mathrm{bd}(A)}(f)$, are sums of covectors in $W F\left(\chi_{A \times \mathbb{R}}\right)$ and covectors in $C \circ \mathrm{WF}(f)$ that have the same base points. The only way a common base point occurs is when

(i) $\omega \in \operatorname{bd}(A)$ and there is a $y \in H(\omega, 0)$ with $(\omega, y \mathrm{~d} \omega) \in \mathrm{WF}\left(\chi_{A}\right)$, generating singularities in $\mathrm{WF}\left(\chi_{A \times \mathbb{R}}\right)$, and

(ii) there is an $x \in \mathbb{R}^{n}$ and $\alpha \neq 0$ so that $(x, \alpha \omega \mathrm{d} x) \in \mathrm{WF}(f)$, generating singularities in $C \circ \mathrm{WF}(f)$.

In this case, the common base point is $(\omega, x \cdot \omega)$. Since $\chi_{A}$ is a real function, if $(\omega, y \mathrm{~d} \omega) \in \operatorname{WF}\left(\chi_{A}\right)$, then so is $(\omega, t y \mathrm{~d} \omega)$ for any $t \neq 0$. Therefore, corresponding covectors for (i) in $\mathrm{WF}\left(\chi_{A \times \mathbb{R}}\right)$ are given by

$$
\left\{(\omega, x \cdot \omega ; t y \mathrm{~d} \omega+0 \mathrm{~d} s): t \neq 0,(\omega, y \mathrm{~d} \omega) \in \mathrm{WF}\left(\chi_{A}\right)\right\}
$$

Similarly, the corresponding covectors for (ii) in $C \circ \mathrm{WF}(f)$ are

$$
\left\{\left(\omega, x \cdot \omega ; t^{\prime} \alpha\left(-\pi_{\omega}(x) \mathrm{d} \omega+\mathrm{d} s\right)\right): t^{\prime}>0\right\}
$$

( $f$ is not assumed to be real, so $\mathrm{WF}(f)$ is only positive homogenous in the cotangent coordinate).

Adding covectors in (45) and (46) gives covectors in $W_{\mathrm{bd}(A)}(f)$. Putting all this together,

$$
\begin{gathered}
W(\omega, x, \alpha):=\left\{\left(\omega, x \cdot \omega ;-\alpha t^{\prime}\left(\left[\pi_{\omega}(x)+t y\right] \mathrm{d} \omega+\mathrm{d} s\right)\right):\right. \\
\left.t^{\prime}>0, t \neq 0,(\omega, y \mathrm{~d} \omega) \in \mathrm{WF}\left(\chi_{A}\right)\right\}
\end{gathered}
$$

is the subset of $W_{\mathrm{bd}(A)}(f)$ associated to $\omega \in \mathrm{bd}(A)$ and each $\alpha \neq 0$ and each $x \in \mathbb{R}^{n}$ such that $(x, \alpha \omega \mathrm{d} x) \in \mathrm{WF}(f)$. Note that we have rescaled $t$ in order to factor as indicated in (47). 
To accomplish the step (e) in our paradigm, we let $P$ be a pseudodifferential operator. Then, by containment (15),

$$
\mathrm{WF}\left(R_{\nu}^{*} P R_{\mu, A} f\right) \subset C^{t} \circ \mathcal{Q}(A \times \mathbb{R}, C \circ \mathrm{WF}(f)) .
$$

We now compute $C^{t} \circ \mathcal{Q}(A \times \mathbb{R}, C \circ \mathrm{WF}(f))$. Using (44) and the composition rules, first observe that

$$
\begin{aligned}
& C^{t} \circ \mathcal{Q}(A \times \mathbb{R}, C \circ \mathrm{WF}(f)) \\
&=C^{t} \circ\left[(C \circ \mathrm{WF}(f)) \cap\left\{((\omega, s), \eta) \in T^{*}(\Xi): \omega \in A\right\}\right] \\
& \cup C^{t} \circ \mathrm{WF}\left(\chi_{A \times \mathbb{R}}\right) \\
& \cup C^{t} \circ W_{\mathrm{bd}(A)}(f) .
\end{aligned}
$$

We examine the three terms in the right side of the equation (48) separately. First, we get

$$
\begin{aligned}
& C^{t} \circ\left[(C \circ \mathrm{WF}(f)) \cap\left\{((\omega, s), \eta) \in T^{*}(\Xi): \omega \in A\right\}\right] \\
& \left.\quad=\left[\left(C^{t} \circ C\right) \circ \mathrm{WF}(f)\right)\right] \cap\left[C^{t} \circ\left\{((\omega, s), \eta) \in T^{*}(\Xi): \omega \in A\right\}\right] .
\end{aligned}
$$

Because $\Pi_{L}$ is injective and $\Pi_{R}$ is surjective to $T^{*}\left(\mathbb{R}^{n}\right) \backslash \mathbf{0}$,

$$
C^{t} \circ C=\Delta:=\left\{(x, \xi \mathrm{d} x ; x, \xi \mathrm{d} x):(x, \xi \mathrm{d} x) \in T^{*} \mathbb{R}^{n} \backslash \mathbf{0}\right\}
$$

and $\Delta \circ \mathrm{WF}(f)=\mathrm{WF}(f)$. Furthermore,

$$
C^{t} \circ\left\{((\omega, s), \eta) \in T^{*}(\Xi): \omega \in A\right\}=\mathcal{V}_{A} .
$$

Hence, the first set in (48) is equal to the set of visible singularities (32)

$$
\mathrm{WF}_{A}(f)=\mathrm{WF}(f) \cap \mathcal{V}_{A} .
$$

For the second set in (48) observe that $C^{t} \circ \mathrm{WF}\left(\chi_{A \times \mathbb{R}}\right)=\emptyset$ since the $\mathrm{d} s$-components of covectors in $\mathrm{WF}\left(\chi_{A \times \mathbb{R}}\right)$ is zero and the $\mathrm{d} s$-components of covectors in $C^{t}$ is always non-zero.

Finally, we consider the set $C^{t} \circ W_{\mathrm{bd}(A)}(f)$. Let $\omega \in \mathrm{bd}(A)$ and let $x \in \mathbb{R}^{n}$ and $\alpha \neq 0$ such that $(x, \alpha \omega \mathrm{d} x) \in \mathrm{WF}(f)$. Then, (47) gives the subset, $W(\omega, x, \alpha)$, of $W_{\mathrm{bd}(A)}(f)$ associated to $\omega, x$, and $\alpha$. For each $y$ such that $(\omega, y \mathrm{~d} \omega) \in \mathrm{WF}\left(\chi_{A}\right)$, there are elements of $W(\omega, x, \alpha)$ for each $t \neq 0$ and $t^{\prime}>0$ :

$$
\gamma=\left(\omega, x \cdot \omega ;-\alpha t^{\prime}\left(\left[\pi_{\omega}(x)+t y\right] \mathrm{d} \omega+\mathrm{d} s\right)\right) .
$$


Then, $C^{t} \circ\{\gamma\}$ is the covector $\left(x^{\prime}, \alpha \omega \mathrm{d} x\right)$, such that $x^{\prime} \in H(\omega, x \cdot \omega)$ (since $\left.x^{\prime} \cdot \omega=x \cdot \omega\right)$ and such that

$$
\pi_{\omega}\left(x^{\prime}\right)=\pi_{\omega}(x)+t y
$$

(see the definition of $C^{t}$ and (25)). Since $x^{\prime} \in H(\omega, x \cdot \omega)$,

$$
x^{\prime}=x+t y
$$

and since $t$ is arbitrary, the set

$$
\left\{(x+t y, \alpha \omega \mathrm{d} x): t \neq 0,(\omega, y \mathrm{~d} \omega) \in \mathrm{WF}\left(\chi_{A}\right)\right\}
$$

is the set of added singularities coming from $\omega \in \mathrm{bd}(A), y \in H(\omega, 0)$ such that $(\omega, y \mathrm{~d} \omega) \in W F\left(\chi_{A}\right)$ and $x \in \mathbb{R}^{n}$ and $\alpha \neq 0$ such that $(x, \alpha \omega \mathrm{d} x) \in$ $\mathrm{WF}(f)$. This proves (33) and (34).

Containment (36) is proven using Theorem 6.3, which is proven below. Let $(x, \xi \mathrm{d} x) \in \mathrm{WF}(f) \cap \mathcal{V}_{\operatorname{int}(A)}$. Then, at least one of the unit vectors $\omega(\xi)$ or $-\omega(\xi)$ (defined in Proposition 5.1) is in $\operatorname{int}(A)$. Without loss of generality, assume $\omega_{0}=\omega(\xi) \in \operatorname{int}(A)$.

First we consider case (i). Let $\varphi$ be a smooth cutoff function in $\omega$ that is supported in a small open set $U \subset A$ and equal to one in a smaller neighborhood $U^{\prime}$ of $\omega_{0}$. Since $\operatorname{cl}(U) \subset A$, if $\omega \in \operatorname{cl}(U)$ then $-\omega \notin \operatorname{cl}(U)$.

We define $\mathcal{K}_{\varphi}$ as the multiplication operator $\mathcal{K}_{\varphi} g(\omega, s)=\varphi(\omega) g(\omega, s)$.

Let

$$
g_{1}=P \mathcal{K}_{\varphi} R_{\mu}(f), \quad g_{2}=P\left[\chi_{A \times \mathbb{R}}-\varphi\right] R_{\mu}(f) .
$$

By Theorem 6.3 part (i) applied to the set $\mathrm{cl}(U)$, the symbol of $R_{\nu}^{*} P \mathcal{K}_{\varphi} R_{\mu}$ is elliptic on $\mathcal{V}_{U}$ and so at $(x, \xi \mathrm{d} x)$. Therefore, $(x, \xi \mathrm{d} x) \in \mathrm{WF}\left(R_{\nu}^{*} g_{1}\right)$.

We now show $(x, \xi \mathrm{d} x) \notin \mathrm{WF}\left(R_{\nu}^{*} g_{2}\right)$. Because $\left[\chi_{A \times \mathbb{R}}-\varphi\right]$ is zero on $U^{\prime} \times$ $\mathbb{R},\left[\chi_{A \times \mathbb{R}}-\varphi\right] R_{\mu} f$ is zero on $U^{\prime} \times \mathbb{R}$. Therefore, $\left.g_{2}=P\left[\chi_{A \times \mathbb{R}}-\varphi\right] R_{\mu}(f)\right)$ is smooth on $U^{\prime} \times \mathbb{R}$. Since $\omega(\xi) \in U^{\prime}, \lambda_{0}(x, \xi) \notin \mathrm{WF}\left(g_{2}\right)$. By the nonsymmetry condition $(35),-\omega(\xi) \notin A$, so $g_{2}$ is zero and hence smooth near $-\omega(\xi)$. This implies that $\lambda_{1}(x, \xi) \notin \mathrm{WF}\left(g_{2}\right)$. Using the Hörmander-Sato Lemma (see (9)) $\mathrm{WF}\left(R_{\nu}^{*} g_{2}\right) \subset C^{t} \circ \mathrm{WF}\left(g_{2}\right)$, so, by (27) the only two covectors, $\lambda_{0}(x, \xi)$ and $\lambda_{1}(x, \xi)$, that can contribute to wavefront of $R_{\nu}^{*} g_{2}$ at $(x, \xi \mathrm{d} x)$ are not in $\mathrm{WF}\left(g_{2}\right)$ so $(x, \xi \mathrm{d} x) \notin \mathrm{WF}\left(R_{\nu}^{*} g_{2}\right)$.

Therefore, $(x, \xi \mathrm{d} x) \in \mathrm{WF}\left(R_{\nu}^{*} g_{1}+R_{\nu}^{*} g_{2}\right)=W F\left(\mathcal{L}_{A} f\right)$, and this proves the final part of the theorem in case (i).

Now we consider case (ii) and note that the symbol of $P$ is of the same $\operatorname{sign}$ on $V_{A}^{R}$. Let $\omega_{0} \in \operatorname{int}(A)$. Then, $-\omega_{0} \in \operatorname{int}(A)$ by the symmetry 
condition for this case. We let $U \subset A$ be a neighborhood of $\omega_{0}$ small enough so that $U$ is disjoint from $(-1) U$. Since $A$ is symmetric, so is $\operatorname{int}(A)$, and $(-1) U \subset \operatorname{int}(A)$. Let $\widetilde{U}=U \cup(-1) U$ and let $\varphi$ be a smooth, nonnegative, even function that is supported in $\widetilde{U}$ and is one in a smaller neighborhood, $U^{\prime}$ of $\omega_{0}$ (and therefore in the neighborhood of $(-1) U^{\prime}$ of $-\omega_{0}$ ). Let $g_{1}$ and $g_{2}$ be as defined in the first part of the proof. By Theorem 6.3 case (ii) applied on $\operatorname{cl}(\widetilde{U})$, the symbol of $R_{\nu}^{*} P \mathcal{K}_{\varphi} R_{\mu}$ is elliptic on $\mathcal{V}_{U}$ and so at $(x, \xi \mathrm{d} x)$. Therefore, $(x, \xi \mathrm{d} x) \in \mathrm{WF}\left(R_{\nu}^{*} g_{1}\right)$.

We now show $(x, \xi \mathrm{d} x) \notin \mathrm{WF}\left(R_{\nu}^{*} g_{2}\right)$. Let $\widetilde{U}^{\prime}=U^{\prime} \cup(-1) U^{\prime}$. Because $\left[\chi_{A \times \mathbb{R}}-\varphi\right]$ is zero on $\widetilde{U}^{\prime} \times \mathbb{R},\left[\chi_{A \times \mathbb{R}}-\varphi\right] R_{\mu} f$ is zero on $\widetilde{U}^{\prime} \times \mathbb{R}$. Therefore, $\left.g_{2}=P\left[\chi_{A \times \mathbb{R}}-\varphi\right] R_{\mu}(f)\right)$ is smooth on $\widetilde{U}^{\prime} \times \mathbb{R}$. Since $\omega(\xi) \in U^{\prime} \subset \widetilde{U}^{\prime}$, $\lambda_{0}(x, \xi) \notin \mathrm{WF}\left(g_{2}\right)$. For the analogous reason, $\lambda_{1}(x, \xi) \notin \mathrm{WF}\left(g_{2}\right)$. The final part of the proof continues as for the case (i) to conclude that $(x, \xi \mathrm{d} x) \in$ $\mathrm{WF}\left(R_{\nu}^{*} g_{1}+R_{\nu}^{*} g_{2}\right)=W F\left(\mathcal{L}_{A} f\right)$.

We now give the proofs of Theorems 6.1-6.3.

Proof of Theorem 6.1. We use the notation, conventions, and symbol calculation in [19, Theorem 3.1]. Recall that $\Pi_{R}: C \rightarrow T^{*}\left(\mathbb{R}^{n}\right) \backslash \mathbf{0}$ and $\Pi_{L}: C \rightarrow T^{*}(\Xi) \backslash \mathbf{0}$ are the natural projections. Let

$$
Z=\left\{(\omega, s, x): \omega \in S^{n-1}, x \in \mathbb{R}^{n}, s=\omega \cdot x\right\},
$$

then $Z$ is the set in over which the Schwartz kernel of $R_{\mu}$ integrates (e.g., [19]). To define the measures used in [19], we define global coordinates for $Z$ :

$$
(\omega, x) \mapsto(\omega, x \cdot \omega, x) .
$$

Of course, $(\omega, s, x)$ are global coordinates on $\Xi \times \mathbb{R}^{n}$. The measure on $Z$ associated to $R_{\mu}$ is $\mu(\omega, x) d \omega d x$ (see equation (16) in [19]). Equation (14) in [19] and the discussion below it give the symbol of $R_{\mu}$ as the half density

$$
\sigma\left(R_{\mu}\right)=\frac{(2 \pi)^{(n-1) / 2} \mu(\omega, x) d \omega d x \sqrt{d s d \eta}}{\sqrt{d \omega d s d x} \Pi_{R}^{*}\left(\left|\sigma_{\mathbb{R}^{n}}\right|\right)}
$$

where $\left|\sigma_{\mathbb{R}^{n}}\right|$ is the density from the canonical symplectic form on $T^{*}\left(\mathbb{R}^{n}\right)$ and $\Pi_{R}^{*}\left(\left|\sigma_{\mathbb{R}^{n}}\right|\right)$ is its pull back to $C$. Finally $\eta$ is the fiber coordinate in the conormal bundle of $Z$. A similar proof shows that the symbol of $R_{\nu}^{*}$ is given by

$$
\sigma\left(R_{\nu}^{*}\right)=\frac{(2 \pi)^{(n-1) / 2} \nu(\omega, x) d \omega d x \sqrt{d s d \eta}}{\sqrt{d \omega d s d x} \Pi_{L}^{*}\left(\left|\sigma_{\Xi}\right|\right)} .
$$


The pseudodifferential operator $\mathrm{PK}_{\varphi}$ has symbol $\varphi(\omega) p(\omega, s, \gamma)$ (where $\gamma \in$ $\left.T_{(\omega, s)}^{*}(\Xi)\right)$ so $P \mathcal{K}_{\varphi} R_{\mu}$ is a standard smooth FIO and its top order symbol is

$$
\sigma\left(P \mathcal{K}_{\varphi} R_{\mu}\right)=\frac{(2 \pi)^{(n-1) / 2} p(\omega, s, \gamma) \varphi(\omega) \mu(\omega, x) d \omega d x \sqrt{d s d \eta}}{\sqrt{d \omega d s d x} \Pi_{R}^{*}\left(\left|\sigma_{\mathbb{R}^{n}}\right|\right)}
$$

when evaluated at covectors on $C$.

Let $(x, \xi \mathrm{d} x) \in T^{*}\left(\mathbb{R}^{n}\right) \backslash \mathbf{0}$. To calculate the symbol of the composition of $R_{\nu}^{*}$ with $P \mathcal{K}_{\varphi} R_{\mu}$ one uses the note at the top of p. 338 of [19]: since the projection $\Pi_{R}: C \rightarrow T^{*}\left(\mathbb{R}^{n}\right) \backslash \mathbf{0}$ is two-to-one, the symbol of $R_{\nu}^{*} P \mathcal{K}_{\varphi} R_{\mu}$ at $(x, \xi \mathrm{d} x) \in T^{*}\left(\mathbb{R}^{n}\right)$ is the sum of the product $\sigma\left(R_{\nu}^{*}\right) \cdot \sigma\left(P \mathcal{K}_{\varphi} R_{\mu}\right)$ at the two preimages. Those preimages are given by $\Pi_{R}^{-1}(x, \xi \mathrm{d} x)$, and by Proposition 5.1 , they are the two covectors $\left(\lambda_{0}(x, \xi) ; x, \xi \mathrm{d} x\right)$ and $\left(\lambda_{1}(x, \xi) ; x, \xi \mathrm{d} x\right)$.

Under the conventions of [19], the symbol of $R_{\nu}^{*} P \mathcal{K}_{\varphi} R_{\mu}$ at $(x, \xi \mathrm{d} x)$ is the sum

$$
\begin{aligned}
\sigma\left(R_{\nu}^{*} P \mathcal{K}_{\varphi} R_{\mu}\right)(x, & \xi \mathrm{d} x)=\left\{\frac{(2 \pi)^{n-1}(d \omega \mathrm{d} x)^{2} d s d \eta}{d \omega d s d x \Pi_{R}^{*}\left(\left|\sigma_{\mathbb{R}^{n}}\right|\right) \Pi_{L}^{*}\left(\left|\sigma_{\Xi}\right|\right)}\right\} \\
\times & {\left[\varphi(\omega(\xi)) \nu(\omega(\xi), x) \mu(\omega(\xi), x) p\left(\lambda_{0}(x, \xi)\right)\right.} \\
& \left.+\varphi(-\omega(\xi)) \nu(-\omega(\xi), x) \mu(-\omega(\xi), x) p\left(\lambda_{1}(x, \xi)\right)\right]
\end{aligned}
$$

Now, [19, Lemma 3.2] shows, for the Radon line transform, that the term on the top right in braces in (53) can be simplified to equal to $(2 \pi)^{n-1} /\|\xi\|^{n-1}$. Putting this into (53) proves the symbol calculation (39).

Proof of Theorem 6.2. Because the symbol of $\mathcal{L}_{\varphi}$ is elliptic on $\mathcal{V}_{\operatorname{int}(A)}$ by assumption, the left hand containment (40) follows from e.g., [22, Prop. $6.9]$.

We now prove that the right-hand containment in (40). First, note that $\mathrm{WF}\left(\mathcal{L}_{\varphi} f\right) \subset \mathrm{WF}(f)$ since $\mathcal{L}_{\varphi}$ is a standard pseudodifferential operator. Second, $\mathcal{L}_{\varphi}$ smooths outside of $\mathcal{V}_{A}$ for the following reason. Since $\varphi$ is zero outside of $\{(\omega, s): \omega \in A\}, \operatorname{WF}\left(\varphi R_{\mu} f\right) \subset V_{A}^{R}$. Now, using (49) and the Hörmander Sato Lemma (9), one shows that $\operatorname{WF}\left(\mathcal{L}_{\varphi} f\right) \subset C^{t} \circ V_{A}^{R}=\mathcal{V}_{A}$. This proves the right hand containment.

Proof of Theorem 6.3. In each case, we will show that $\sigma\left(\mathcal{L}_{\varphi}\right)$ is elliptic on $\mathcal{V}_{\operatorname{int}(A)}$. Let $(x, \xi \mathrm{d} x) \in \mathcal{V}_{\operatorname{int}(A)}$, then either $\omega(\xi)$ or $-\omega(\xi)$ or both are in $\operatorname{int}(A)$. Without loss of generality, we assume $\omega(\xi) \in \operatorname{int}(A)$. Therefore, $\varphi(\omega(\xi)) \neq 0$.

In case (i), we assume $\mu$ and $\nu$ are smooth and nowhere zero, and we assume that, if $\omega(\xi) \in \operatorname{int}(A)$, then $-\omega(\xi) \notin A$. Therefore, one and only one term in brackets in (39) is nonzero, and the symbol is elliptic on $\mathcal{V}_{\operatorname{int}(A)}$. 
In case (ii), we assume $\mu$ and $\nu$ are positive, the top order symbol of $P$, $\sigma(P)=p$, is real, elliptic, and of the same sign everywhere on $V_{A}^{R}$. Since $\varphi \neq 0$ on $\operatorname{int}(A)$ and $\nu \mu>0$, at least the first term in brackets in (39) (the one containing $\omega(\xi))$ is nonzero. The second term (containing $-\omega(\xi))$ either has the same sign as this term (since the sign of $p$ does not change) or is zero (if $\varphi(-\omega(\xi))=0$ ). Therefore the sum is nonzero and so the symbol of $\mathcal{L}_{\varphi}$ is elliptic on $\mathcal{V}_{\operatorname{int}(A)}$.

In either case, we have concluded the symbol of $\mathcal{L}_{\varphi}$ is elliptic on $\mathcal{V}_{\operatorname{int}(A)}$. Now, one can use the conclusion of Theorem 6.2 to finish the proof.

\section{References}

[1] G. Beylkin. The inversion problem and applications of the generalized Radon transform. Comm. Pure Appl. Math., 37:579-599, 1984.

[2] J. Frikel and E. T. Quinto. Characterization and reduction of artifacts in limited angle tomography. Inverse Problems, 29(12):125007, Dec. 2013.

[3] J. Frikel and E. T. Quinto. A paradigm for the characterization of artifacts in tomography. arXiv:1409.4103 [math.AP], 2014. Preprint.

[4] J. Frikel and E. T. Quinto. Artifacts in incomplete data tomography with applications to photoacoustic tomography and sonar. SIAM J. Appl. Math., 75(2):703-725, 2015.

[5] V. Guillemin and S. Sternberg. Geometric Asymptotics. American Mathematical Society, Providence, RI, 1977.

[6] S. Helgason. Integral geometry and Radon transforms. Springer, New York, 2011.

[7] L. Hörmander. Fourier Integral Operators, I. Acta Mathematica, 127:79-183, 1971.

[8] L. Hörmander. The analysis of linear partial differential operators. I. Classics in Mathematics. Springer-Verlag, Berlin, 2003. Distribution theory and Fourier analysis, Reprint of the second (1990) edition [Springer, Berlin].

[9] A. Katsevich. Local tomography for the limited-angle problem. $J$. Math. Anal. Appl., 213(1):160-182, 1997. 
[10] P. Kuchment, K. Lancaster, and L. Mogilevskaya. On local tomography. Inverse Problems, 11:571-589, 1995.

[11] A. K. Louis. Incomplete data problems in X-ray computerized tomography I. Singular value decomposition of the limited angle transform. Numerische Mathematik, 48:251-262, 1986.

[12] F. Natterer. The mathematics of computerized tomography. B. G. Teubner, Stuttgart, 1986.

[13] F. Natterer and F. Wübbeling. Mathematical methods in image reconstruction. SIAM Monographs on Mathematical Modeling and Computation. Society for Industrial and Applied Mathematics (SIAM), Philadelphia, PA, 2001.

[14] L. V. Nguyen. How strong are streak artifacts in limited angle computed tomography? Inverse Problems, 31(5):055003, 26, 2015.

[15] L. V. Nguyen. On artifacts in limited data spherical radon transform: flat observation surfaces. SIAM J. Math. Anal., 47(4):2984-3004, 2015.

[16] R. G. Novikov. Une formule d'inversion pour la transformation d'un rayonnement X atténué. C. R. Acad. Sci. Paris Sér. I Math., 332(12):1059-1063, 2001.

[17] V. Palamodov. Reconstruction from Limited Data of Arc Means. J. Fourier Anal. Appl., 6:25-42, 2000.

[18] X. Pan, E. Y. Sidky, and M. Vannier. Why do commercial CT scanners still employ traditional, filtered back-projection for image reconstruction? Inverse Problems, 25(12):123009, 2009.

[19] E. T. Quinto. The dependence of the generalized Radon transform on defining measures. Trans. Amer. Math. Soc., 257:331-346, 1980.

[20] E. T. Quinto. Singularities of the X-ray transform and limited data tomography in $\mathbb{R}^{2}$ and $\mathbb{R}^{3}$. SIAM J. Math. Anal., 24(5):1215-1225, 1993.

[21] W. Rudin. Functional analysis. McGraw-Hill Book Co., New York, 1973. McGraw-Hill Series in Higher Mathematics.

[22] F. Trèves. Introduction to Pseudodifferential and Fourier Integral Operators. Volume 2: Fourier Integral Operators. Plenum Press, New York and London, 1980. 